\title{
Impacto de los Inputs de Marcos de Ventanas en EnergyPlus en la Estimación de Eficiencia Energética de Edificios
}

\section{Impact of Window Frames Inputs in EnergyPlus on the Estimation of Building Energy Efficiency}

Maureen de Gastines * Laboratorio de Ambiente Humano y Vivienda (LAHV. CONICET mdegastines@mendoza-conicet.gob.ar

Andrea Pattini ** Laboratorio de Ambiente Humano y Vivienda (LAHV). CONICET 


\section{Resumen}

El diseño de edificios sustentables hace uso de herramientas de simulación energética como EnergyPlus. Un elemento clave en la eficiencia energética es la ventana, y particularmente el marco, debido a su transmitancia térmica generalmente elevada en relación a los elementos que componen la envolvente edilicia. Además, el diseño de marco reduce la superficie vidriada, disminuyendo el recurso solar aprovechable por ganancia directa. Por lo tanto, cuando se realice una simulación en EnergyPlus, resulta fundamental la adecuada definición del diseño de ventanas a través de los elementos de entrada (inputs). Este trabajo analiza las distintas opciones posibles para la modelización de los marcos de ventanas en EnergyPlus, y los compara entre sí mediante simulación. Los resultados evidencian la elevada sensibilidad de los datos de salida (outputs) frente a la falta de precisión posible en la carga de inputs de marco, resaltando los riesgos de impericia por parte de los profesionales proyectistas.

Palabras clave: Marcos de ventana; EnergyPlus; simulación energética

\section{Abstract}

The design of sustainable buildings makes use of energy simulation tools like EnergyPlus. A key element in energy efficiency is the window, and more specifically the window frame, since it generally has a higher thermal transmittance than the other building envelop components. Moreover, the frame reduces the glazing area, decreasing the solar gain. Therefore, when carrying out a simulation in EnergyPlus, it is essential to specify the design of window frames through the correct definition of inputs. In this paper, the different possible options for the modeling of window frames in EnergyPlus are analyzed, then compared with each other through simulations. The results show that the outputs are very sensitive to the inputs lack of accuracy, which highlights the necessity for building designer professionals to be cautious about those aspects in order to obtain reliable simulation results.

Keywords: Window frame, EnergyPlus, Energy simulation 


\section{Introducción}

Las ventanas son elementos clave en la eficiencia energética de los edificios, debido a que a través de ellas ocurren múltiples intercambios lumínicos y energéticos. En particular, el marco juega un papel significativo en el balance energético asociado a las ventanas, debido a su transmitancia térmica generalmente elevada en relación a los elementos que componen la envolvente edilicia. Además, el diseño de marco reduce la superficie vidriada, disminuyendo el recurso solar aprovechable por ganancia directa.

EnergyPlus es un programa de simulación energética de edificios, que funciona en régimen dinámico y constituye una herramienta ampliamente usada para el análisis energético y diseño de edificios sustentables. El usuario carga los datos de entrada (inputs) del proyecto bajo estudio, y define un archivo climático para luego correr la simulación y obtener la información deseada a través de los datos de salida (outputs). El modelado de edificios implica simplificaciones en la descripción morfológica de los espacios y elementos constituyentes, pero las mismas no deben impactar negativamente sobre la precisión de los outputs. Asimismo, el programa ofrece distintas opciones de modelado para un mismo elemento de la envolvente.

En el caso de las ventanas, un panorama general de las opciones de modelado asociadas a la superficie vidriada en EnergyPlus está dado por Winkelmann (2001). Se distinguen dos posibilidades de modelización de los vidriados, uno usa los datos espectrales completos (Full Spectral Model) y el otro los datos espectrales promediados para cada capa vidriada (Spectral Average Model). Arasteh et al. (2009) desarrollaron luego un modelo simplificado Ilamado Simple Window Model, el cual permite simular los impactos energéticos de las ventanas a partir de los índices térmicos de las mismas (Factor K, Factor solar, Transmitancia visible). El impacto de los inputs relacionados con la parte vidriada de las ventanas fue analizado por Lyons et al. (2010), quienes compararon los tres modelos anteriormente nombrados: el Simple Window Model propuesto por Arasteh et al. versus la modelización más explícita de la ventana (Full Spectral Model y Spectral Average Model). Si bien el modelo simplificado no es tan preciso como el Full Spectral Model, demuestra dar mejores resultados que el Spectral Average Model. Los autores observan que en el caso del simple vidriado el modelo simplificado predice un 6\% más de cargas térmicas aproximadamente que el Full Spectral Model. En el caso del DVH, predice entre un $8 \%$ menos y un $2 \%$ más cargas aproximadamente. Por lo tanto se puede decir que para el uso de SV y DVH clear float, el modelo simplificado constituye una aproximación válida en cuanto al vidriado. Sin embargo, Lam et al. (2014) realizaron la comparación de 6 modelizaciones distintas del vidriado (las 3 anteriores, el método que utiliza datos generados por el programa WINDOW, el método BSDF - Bi-directional Scattering Distribution Functions Method - y el método REM - Refraction Extinction Method) aplicadas a 15 vidriados (sin marco) y llegaron a conclusiones contrarias. Observan que el método Spectral Average Model da resultados muy parecidos al Full Spectral Model (recomendado por EnergyPlus), mientras que el método Simple Window Model tiende a sobreestimar las cargas de refrigeración.

Muchas veces por razones de simplificación los proyectistas simulan las aberturas sin el marco. (Balter et al., 2012; Barea, 2011). Sin embargo, la contribución del marco al balance energético de la ventana no se puede despreciar, debido a que suelen tener una transmitancia térmica muy alta (por lo menos en Argentina), y que los marcos ocupan un porcentaje importante de la superficie de la ventana - del orden del 30\% (de Gastines et al., 2015) lo cual limita la ganancia solar directa.

Los marcos pueden tener geometrías complejas, por lo tanto se necesitan herramientas de simulación por el método de los elementos finitos para determinar sus índices térmicos. EnergyPlus no permite realizar este cálculo, y los valores de transmitancia térmica (K) 
indicados en IRAM 11507-4 no diferencian los distintos diseños y tipologías de marcos. Tampoco se dispone de los valores de factor solar (FS), ya que aunque este valor puede ser obtenido fácilmente para el vidriado, hay que tomar en cuenta la contribución del marco al FS de la ventana completa. Por lo tanto es importante caracterizar correctamente el comportamiento térmico de los marcos por otro medio, además de sus características dimensionales, para que EnergyPlus pueda tomar en cuenta la geometría proyectante, la conductancia a veces muy elevada, además de la reducción de la superficie vidriada propia a cada marco.

Los objetivos específicos del presente trabajo son los siguientes:

1- Analizar las distintas opciones posibles e inputs asociados para la modelización de los marcos de ventanas en EnergyPlus.

2- Caracterizar los índices térmicos (K y FS) de dos modelos de ventanas típicas del país.

3- Cuantificar el impacto de las distintas opciones de inputs de marco analizadas sobre la estimación de eficiencia energética de una vivienda.

\section{Metodología}

Se comparan las distintas opciones posibles para la modelización de los marcos de ventanas en EnergyPlus mediante la simulación de una vivienda simplificada con ventanas corredizas de aluminio sin ruptura de puente térmico.

\section{Modelización de la vivienda de estudio}

Se definió una vivienda simplificada con el objetivo de analizar los impactos de los inputs de marcos sobre las cargas térmicas de dicha vivienda. La misma se compone de una planta rectangular de $8 \times 12 \mathrm{~m}$, sin muros interiores, con paredes de ladrillo común aislado con poliestireno expandido y revocado, piso de hormigón con revestimiento de madera y techo de hormigón aislado y cubierto con baldosas. Los datos de los distintos materiales empleados se muestran en la tabla 1 (Alchapar et al., 2012; Balter et al., 2012; Monroy, 1995).

En cuanto a las aberturas, se seleccionó una tipología de ventanas de uso frecuente en el país: corrediza de aluminio, de dimensiones 1,50 x 1,10 m. Se estudiaron dos tipos de vidriados: simple vidrio (SV) claro de $3 \mathrm{~mm}$, y doble vidriado hermético (DVH) compuesto por dos vidrios claros de $4 \mathrm{~mm}$ separados por una capa de aire

Tabla 1. Composición de los elementos constructivos y propiedades térmicas de los materiales

\begin{tabular}{|c|c|c|c|c|c|c|c|}
\hline $\begin{array}{l}\text { Elementos } \\
\text { constructivos }\end{array}$ & $\begin{array}{c}\text { Rugosidad } \\
- \\
\end{array}$ & $\begin{array}{c}\text { Espesor } \\
\mathrm{m}\end{array}$ & $\begin{array}{c}\text { Conductividad } \\
\qquad \mathrm{W} / \mathrm{mK}\end{array}$ & $\begin{array}{c}\text { Densidad } \\
\text { kg/m3 }\end{array}$ & $\begin{array}{l}\text { Calor } \\
\text { específ. } \\
\mathrm{J} / \mathrm{kgK}\end{array}$ & $\begin{array}{c}\text { Absort. } \\
\text { térmica } \\
-\end{array}$ & $\begin{array}{c}\text { Absor. } \\
\text { solar } \\
-\end{array}$ \\
\hline \multicolumn{8}{|c|}{ Paredes verticales } \\
\hline Revoque & Muy rug. & 0,03 & 0,9 & 1900 & 1000 & 0,9 & 0,3 \\
\hline EPS & & 0,03 & 0 & 15 & 1420 & & \\
\hline Ladrillo & & 0,18 & 0,7 & 1700 & 800 & & \\
\hline Revoque & Muy rug. & 0,03 & 0,9 & 1900 & 1000 & 0,9 & 0,3 \\
\hline \multicolumn{8}{|l|}{ Piso } \\
\hline Tierra & Rugoso & 1 & 1,8 & 1800 & 920 & 0,88 & 0,7 \\
\hline Losa H으음 & & 0,12 & 1,6 & 2400 & 805 & & \\
\hline Piso madera & Suave & 0,03 & 0,2 & 500 & 2800 & 0,6 & 0,9 \\
\hline \multicolumn{8}{|l|}{ Techo } \\
\hline Baldosa & Rugoso & 0,01 & 1,2 & 2100 & 780 & 0,95 & 0,87 \\
\hline EPS & & 0,03 & 0 & 15 & 1420 & & \\
\hline Losa $\mathrm{H} \cong \mathrm{A} \cong$ & & 0,12 & 1,6 & 2400 & 805 & & \\
\hline Estuco yeso & Suave & 0,03 & 0,5 & 741 & 836 & 0,9 & 0,2 \\
\hline
\end{tabular}

Fuente: Elaboración propia 
de $9 \mathrm{~mm}$. Se ubicó mayor superficie vidriada al norte para considerar un caso de vivienda que aprovechara la máxima ganancia de calor directa que provee esta orientación durante el invierno: tres ventanas al norte $(37,5 \%$ de la superficie total de aberturas), dos al oeste y al este $(25 \%)$ y una al sur (12,5\%).

Índices térmicos de las ventanas

WINDOW 6.3 y THERM 6.3 son dos software del Lawrence Berkeley National Laboratory (LBNL) que permiten simular con precisión las ventanas y particularmente los marcos a través del método de los elementos finitos. Se simulan las ventanas de acuerdo al procedimiento NFRC (NFRC 100-2004), basado en la norma ISO 15099 (ISO 15099:2003). Si bien las condiciones de borde estipuladas por la NFRC no son parecidas a las condiciones típicas de temperatura y velocidad de viento que pueden ocurrir en Argentina, impactando negativamente en el

Figura 1a. Sección del umbral con SV y hoja interna

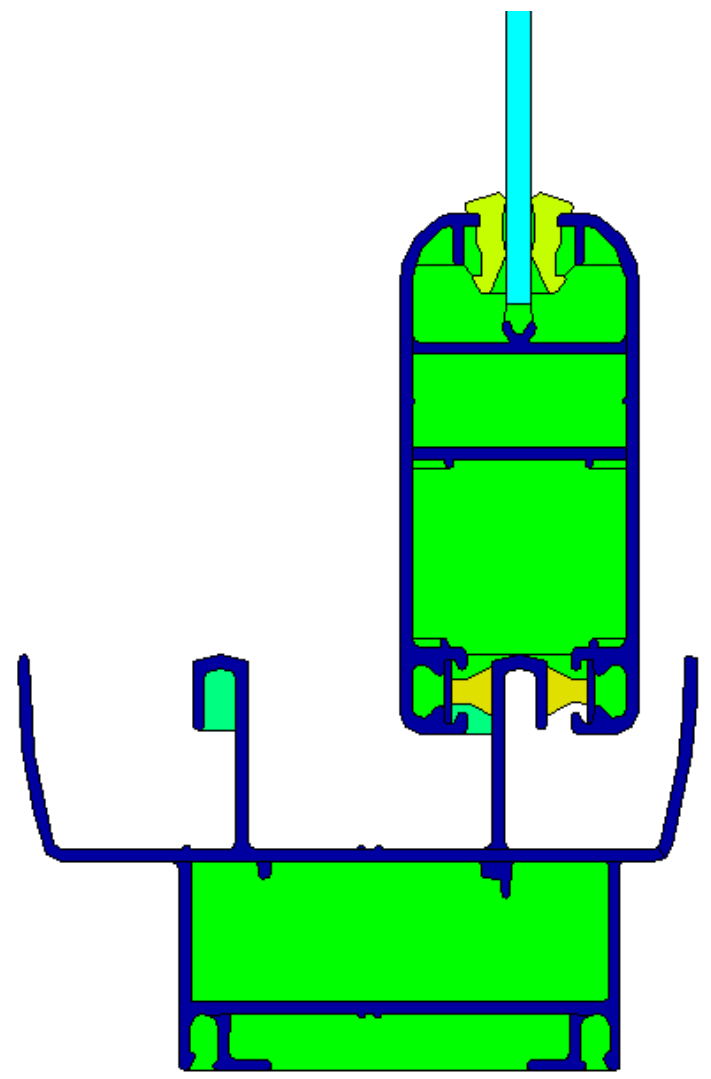

Fuente: Elaboración propia valor de transmitancia térmica resultante (de Gastines et al., 2014), el programa EnergyPlus utiliza los valores así calculados para evaluar la conductancia del marco y así estimar el balance energético a través del marco en cada iteración en función de las condiciones climáticas.

Las figuras $1 \mathrm{a}$ y $1 \mathrm{~b}$ muestran los dibujos en THERM de la sección del umbral de la carpintería seleccionada para el estudio, por un lado con hoja interna y simple vidriado, y por otro caso con hoja externa, en el caso del doble vidriado hermético. Las cavidades internas y parcialmente ventiladas están representadas en verde. La transmitancia térmica de la ventana completa se calcula como el promedio ponderado de los factores $\mathrm{K}$ asociados al centro del vidrio, al borde del vidriado y al marco, por sus áreas respectivas. En cuanto al factor solar, se indicó el valor de FSv y el valor asociado a la ventana completa arrojado por el programa WINDOW. Los índices térmicos K y FS obteni-

Figura 1b. Sección del umbral con DVH y hoja externa.

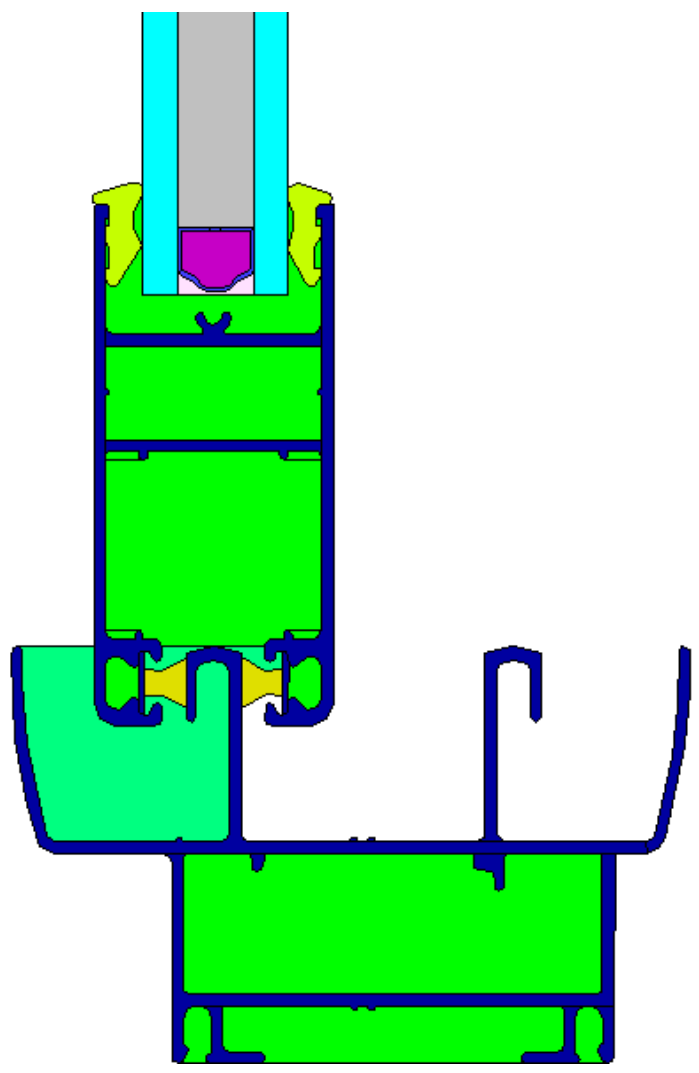

Fuente: Elaboración propia 
Tabla 2. Índices térmicos de las ventanas, en el caso a) con SV y b) con DVH.

\begin{tabular}{|l|c|c|c|}
\hline \multicolumn{1}{|c|}{ Índice } & Símbolo & SV claro $\mathbf{3 m m}$ & DVH claro 4/9/4 \\
\hline Transmitancia térmica del centro del vidriado & $\mathrm{KV}$ & 5,92 & 3,23 \\
\hline Factor Solar del vidriado & $\mathrm{FSV}$ & 0,86 & 0,75 \\
\hline Transmitancia térmica del marco & $\mathrm{Km}$ & 9,96 & 9,94 \\
\hline Transmitancia térmica del borde del vidriado & $\mathrm{Kb}$ & 5,60 & 3,34 \\
\hline Área transparente & $\mathrm{Av}$ & 1,10 & 1,10 \\
\hline Área del marco & $\mathrm{Am}$ & $\mathbf{0 , 5 5}$ & 0,55 \\
\hline Transmitancia térmica de la ventana & $\mathbf{K}$ & $\mathbf{7 , 2 1}$ & $\mathbf{5 , 5 1}$ \\
\hline Factor Solar de la ventana & $\mathbf{F S}$ & $\mathbf{0 , 6 0}$ & $\mathbf{0 , 5 3}$ \\
\hline
\end{tabular}

Fuente: Elaboración propia

dos para estas carpinterías se muestran en la Tabla 2.

Datos climáticos

Se realizaron las simulaciones en EnergyPlus de las cargas térmicas de la vivienda de estudio para seis ciudades de Argentina de climas diferentes: Buenos Aires, Córdoba, Mendoza Salta, Resistencia y Comodoro Rivadavia. Se usaron archivos climáticos TMY ("Typical Meteorological Year") de Weather Analytics.

Comparación de las cargas térmicas para 4 opciones de modelización:

1) Modelado sin marco

En este caso se simuló la ventana solamente con el vidriado. Se utilizó el modelo Full Spectral Data

Figura 2a. Datos espectrales del vidrio claro de $3 \mathrm{~mm}$.

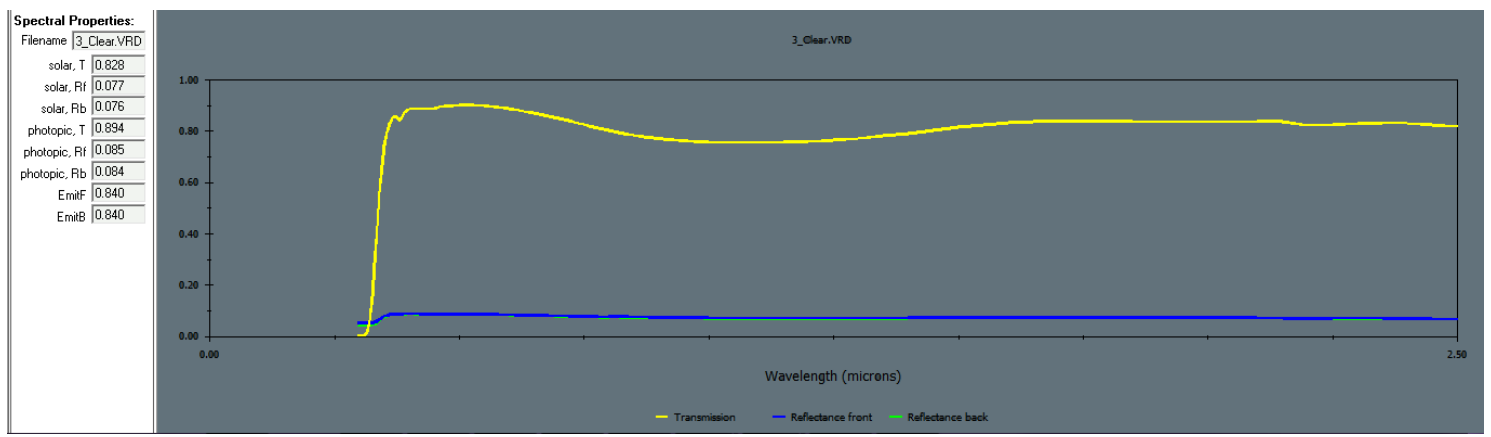

Fuente : Biblioteca Optics6

Figura: Datos espectrales del vidrio claro de $4 \mathrm{~mm}$

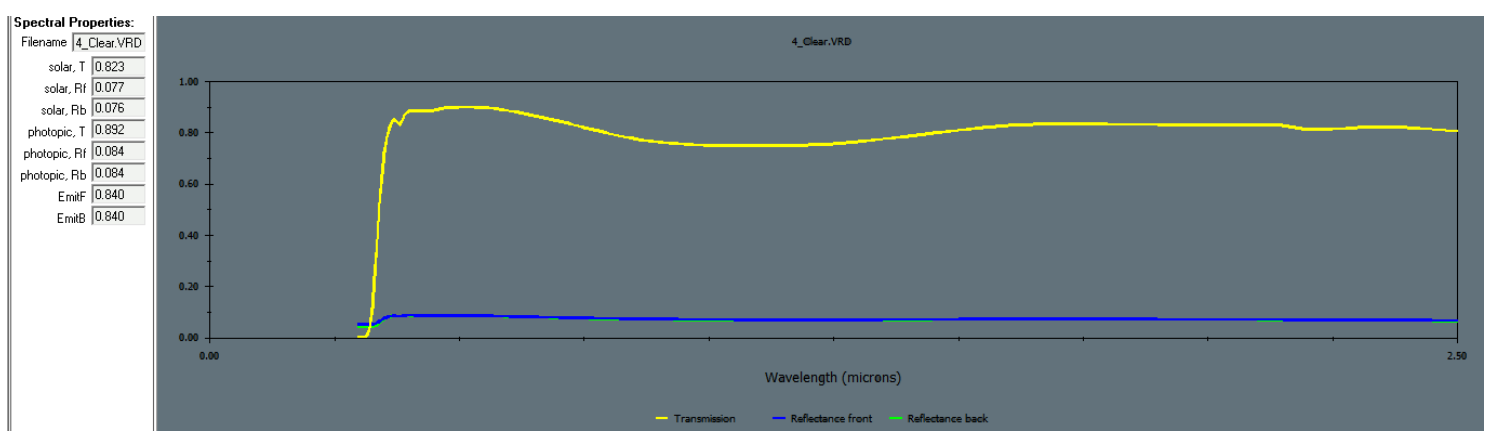

Fuente : Biblioteca Optics6 
(a partir de la base de datos de vidrios de Optics). Las figuras $2 \mathrm{a}$ y $2 \mathrm{~b}$ muestran los datos espectrales de los vidrios claros de $3 \mathrm{~mm}$ y de $4 \mathrm{~mm}$.

2) Modelado con área vidriada reducida

En este caso se simula la ventana sin marco, pero con dimensiones reducidas (es decir, considerando el área real del vidrio) para tener en cuenta la disminución de la ganancia solar debida a la presencia del marco. De esta manera, se puede analizar el efecto del marco sobre las cargas térmicas del edificio.

3) Modelo simplificado de ventana

Este modelo de acuerdo a Arasteh et al. (2009) puede aplicarse a vidriados sin marco, o al conjunto vidriado/marco asimilado a una capa equi- valente sin marco (entrando los valores de $\mathrm{K}$ y FS de la ventana completa). Es útil cuando solo se conocen los índices térmicos de la ventana, sin disponer de suficiente información para describir el marco más detalladamente. A partir de los índices térmicos calculados en WINDOW y THERM, se crea un objeto ventana ("Simple Glazing System") con los valores de K y FS encontrados. El modelo no acepta valores de transmitancia térmica superiores a $7 \mathrm{~W} / \mathrm{m} 2 \mathrm{~K}$, por lo tanto se ingresa este valor en vez del K calculado para la ventana con simple vidrio.

4) Modelo detallado: cargando datos de marco desde WINDOW/THERM

Es posible importar a EnergyPlus los índices térmicos previamente calculados mediante los simuladores WINDOW y THERM, para un mo-

Figura 3. Inputs para la carga de datos de entrada del marco.

\begin{tabular}{|l|l|l|}
\hline Field & Units & Obj1 \\
\hline Name & & Marco \\
\hline Frame Width & $\mathrm{m}$ & 0.103 \\
\hline Frame Outside Projection & $\mathrm{m}$ & 0.038 \\
\hline Frame Inside Projection & $\mathrm{m}$ & 0.038 \\
\hline Frame Conductance & W/m2-K & 500 \\
\hline Ratio of Frame-Edge Glass Conductance to Center-DF-E & & 1 \\
\hline Frame Solar Absorptance & & 0.7 \\
\hline Frame Visible Absorptance & & 0.9 \\
\hline Frame Thermal Hemispherical Emissivity & & 0.9 \\
\hline Divider Type & & DividedLite \\
\hline Divider Width & $\mathrm{m}$ & 0.08 \\
\hline Number of Horizontal Dividers & & 0 \\
\hline Number of Vertical Dividers & & 1 \\
\hline Divider Outside Projection & $\mathrm{m}$ & 0.013 \\
\hline Divider Inside Projection & $\mathrm{m}$ & 0.013 \\
\hline Divider Conductance & W/m2-K & 500 \\
\hline Ratio of Divider-Edge Glass Conductance to Center-0f- & & 1 \\
\hline Divider Solar Absorptance & & 0.7 \\
\hline Divider Visible Absorptance & & 0.9 \\
\hline Divider Thermal Hemispherical Emissivity & & 0.9 \\
\hline
\end{tabular}

\begin{tabular}{|c|c|c|c|c|}
\hline Field & Units & Obji1 & Obi2 & Obj3 \\
\hline Name & & NORTH W/ 3 & NORTH WI 1 & WEST W/ 2 \\
\hline Surface Type & & Window & Window & Window \\
\hline Construction Name & & VENTANA SV $3 \mathrm{~mm}$ & VENTANA SV $3 \mathrm{~mm}$ & VENTANA SV $3 \mathrm{~mm}$ \\
\hline Building Surface Name & & SURFACE NORTH & SURFACE NORTH & SURFACE WEST \\
\hline \multicolumn{5}{|c|}{ Outside Boundary Condition Dbject } \\
\hline View Factor to Ground & & autocalculate & autocalculate & autocalculate \\
\hline \multicolumn{5}{|l|}{ Shading Control Name } \\
\hline Frame and Divider Name & & Marco & Marco & Marco \\
\hline Multiplier & & 1 & 1 & 1 \\
\hline Number of Vertices & & 4 & 4 & 4 \\
\hline Vertex $1 \mathrm{X}$-coordinate & $\mathrm{m}$ & 2.75 & 7.75 & 0 \\
\hline Vertex $1 Y$-coordinate & $\mathrm{m}$ & 8 & 8 & 5.5 \\
\hline
\end{tabular}

Fuente : Elaboración propia 
delado más preciso de las aberturas. En este caso se crea un objeto "Frame and divider" en el cual se indican el ancho del marco, las proyecciones interior y exterior del mismo, el valor de conductancia térmica (es decir el coeficiente de pérdidas sin los coeficientes peliculares), la proporción entre los valores de conductancia del borde del vidrio y del centro del vidrio, y datos de absortancia y emisividad del marco. En el caso de ventanas con divisores (por ejemplo en el caso de un vidrio repartido) se deben ingresar también los mismos datos para ellos.

La figura 3 muestra los inputs obtenidos de la simulación en WINDOW y THERM de la ventana estudiada. (Sección WindowProperty: FrameAndDivider). Se consideró la sección de encuentro del marco corredizo como un divisor, para tomar en cuenta la superficie opaca correspondiente así como el efecto de sombreado resultante.

\section{Resultados}

En la figura 4 están graficadas las demandas de energía para calefacción y refrigeración en los cuatro casos estudiados, para simple vidriado. Estas varían según la localidad entre 10,8 y $114,5 \mathrm{kWh} / \mathrm{m} 2$ para calefacción y entre 13,4 y 107,1 kWh/m2 para refrigeración. La figura 5 indica las diferencias porcentuales obtenidas respecto del caso con marcos. Se observa que para todas las ciudades consideradas, la simulación sin marcos conduce a una reducción de las cargas de calefacción (-8\% a $-22 \%)$ y un aumento de las cargas de refrigeración obtenidas (5\% a 20\%). En cuanto al modelo simplificado y al modelo con área vidriada reducida, se observa una buena adecuación con el modelado más detallado (con marco) para la demanda de calefacción (menos de 2,5\% de diferencia). Sin embargo ambos modelos subestiman las cargas de refrigeración respecto de los resultados obtenidos a través del modelado con marcos ( $-5 \%$ a $-15 \%$ para el modelo simplificado, $-13 \%$ a -31\% para el modelo con área vidriada reducida).

Figura 4. Demandas de energía (caso SV).

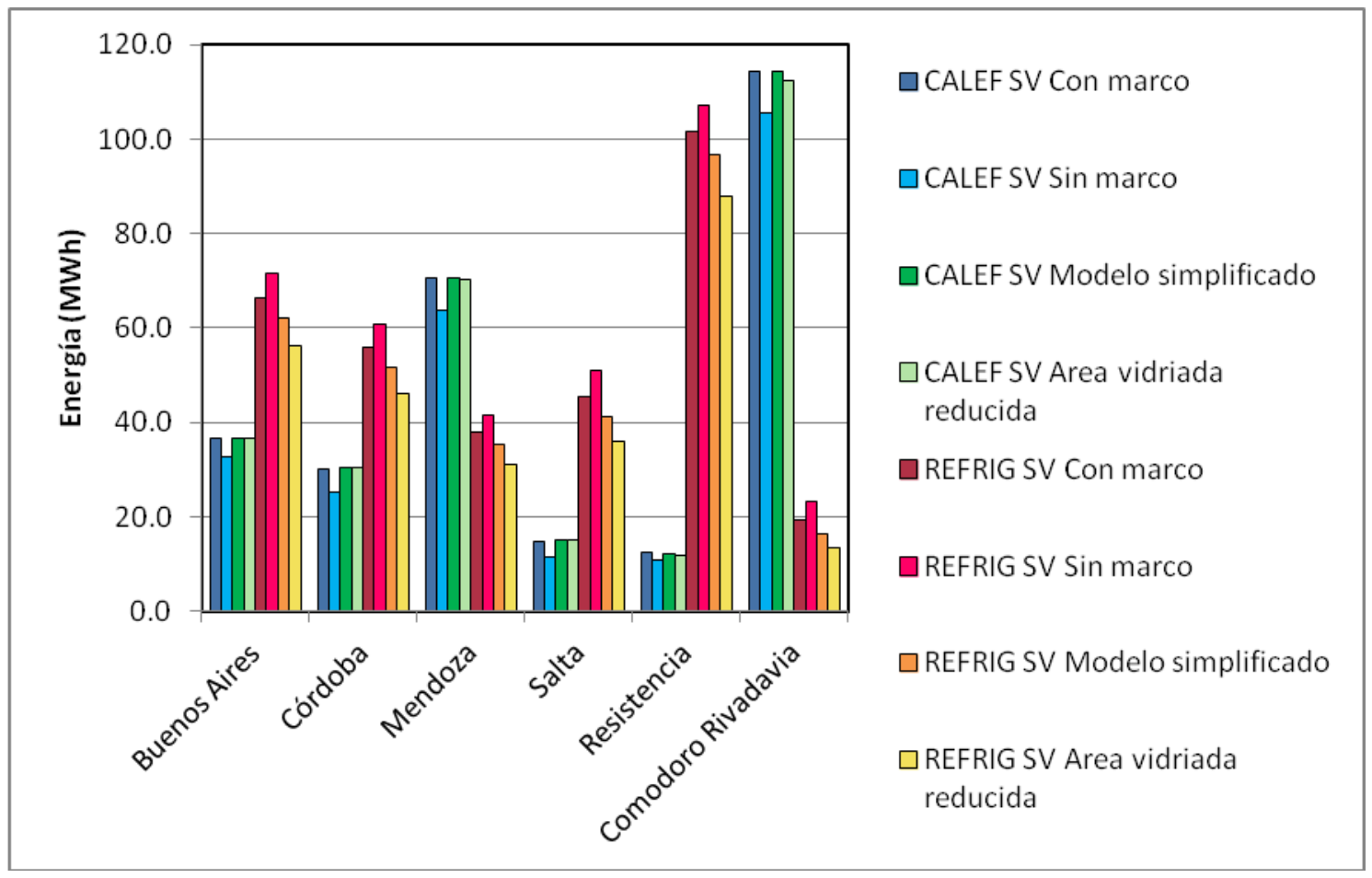

Fuente : Elaboración propia 


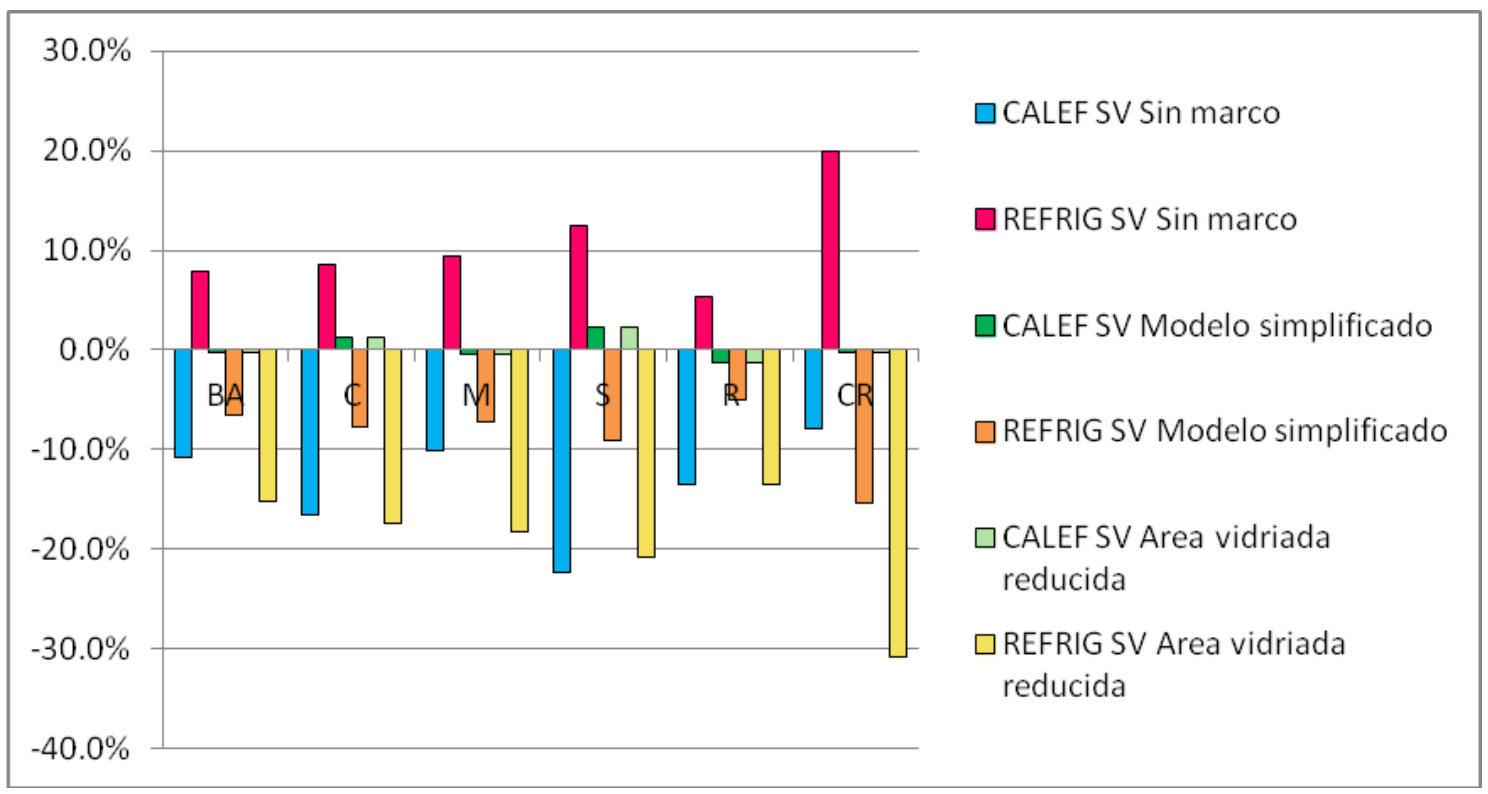

Fuente: Elaboración propia

Observando ahora los resultados obtenidos considerando la utilización del Doble Vidriado Hermético (figuras 6 y 7 ), se constata que las demandas varían entre 9,9 y 116,5 kWh/ m2 para calefacción y entre 12,3 y 101,2 kWh/ m2 para refrigeración. Al igual que el caso con simple vidriado, la simulación sin marcos conduce a una reducción de las cargas de calefacción (-8\% a -22\%) y un aumento de las cargas de refrigeración obtenidas (5\% a 19\%). En cuanto al modelo simplificado, se observa una sobreestimación de la demanda de calefacción (7\% a 23\%) y una subestimación de las cargas de refrigeración (-5\% a -22\%) con respecto a los resultados obtenidos a través del modelado con marcos. Finalmente, el modelo con área vidriada reducida presenta una relativamente buena adecuación con respecto a las cargas térmicas de calefacción (menos de 6\%, excepto para Salta) pero subestima de manera importante las cargas térmicas de refrigeración (-13\% a -32\% de diferencia).

De los resultados previamente expuestos, se destaca que para todas las localidades seleccionadas, los cuatro métodos de modelado de las ventanas bajo estudio producen diferencias significativas en las demandas de calefacción y refrigeración obtenidas. En el caso del método simplificado, se deben a que la información cargada en los inputs es global a nivel de la ventana, por lo tanto el programa no puede adivinar adecuadamente cuáles son el área vidriada y las propiedades térmicas de cada parte de la ventana. En el caso del método sin marco, al sobreestimar la superficie transparente aumenta la ganancia solar y por lo tanto crece la demanda de refrigeración y disminuye la demanda de calefacción. Finalmente, el método del área vidriada reducida estima mejor la ganancia solar pero asocia al área ocupada por el marco una transmitancia térmica insuficiente (la del muro en vez de la transmitancia elevada del marco), además de una absortancia solar reducida (0,3 contra 0,9 para el marco de aluminio). Estos dos aspectos se compensan en invierno y se suman en verano, por eso el error es más significativo para las cargas de refrigeración que para las cargas de calefacción.

Observando más en detalle los casos de Buenos Aires y Mendoza y enfocándose a los métodos con y sin marco, se comparan las demandas en calefacción y refrigeración para ambos métodos y para los dos tipos de vidriado estu- 


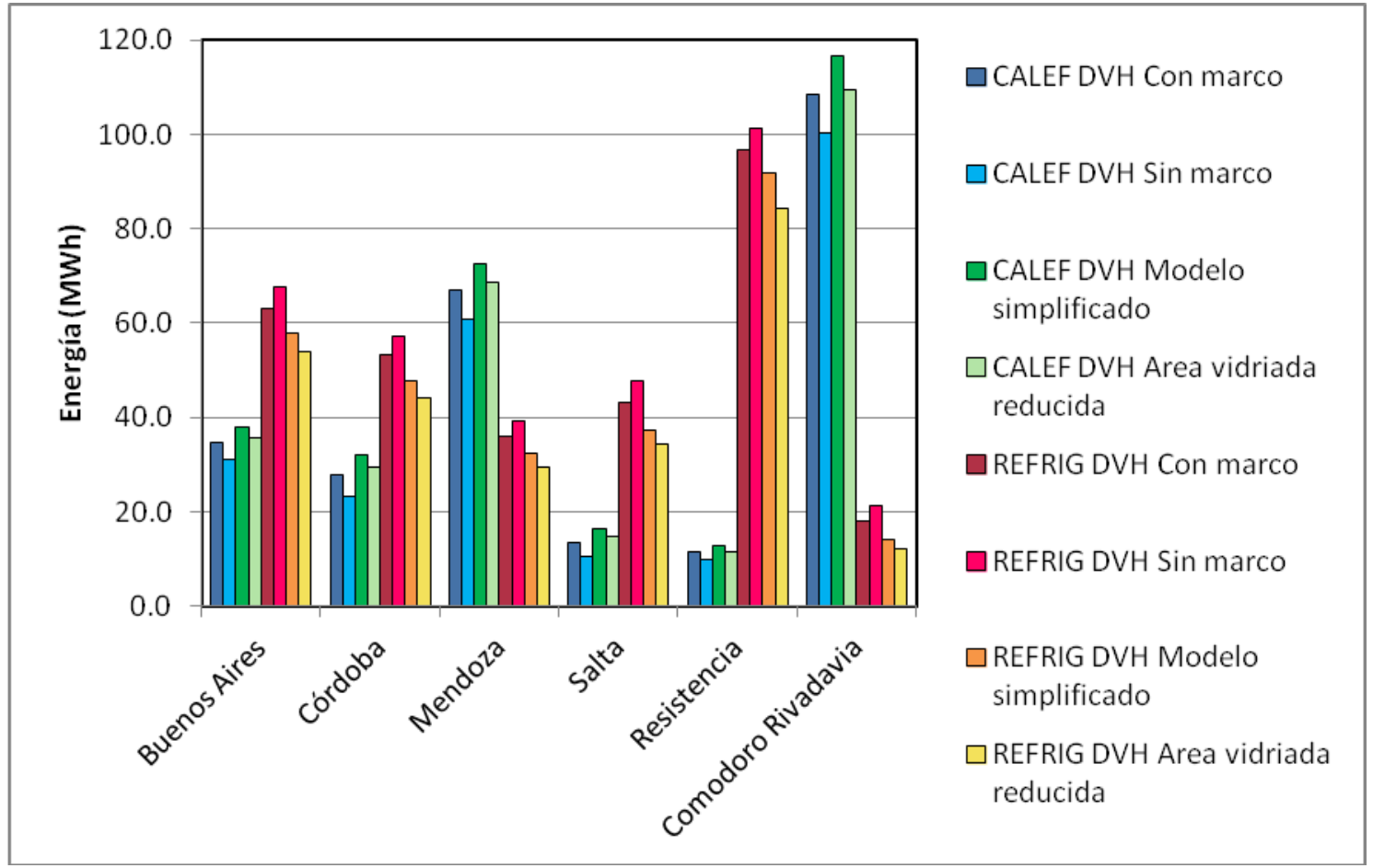

Fuente : Elaboración propia

Figura 7. Diferencias porcentuales respecto del modelo detallado (caso DVH).

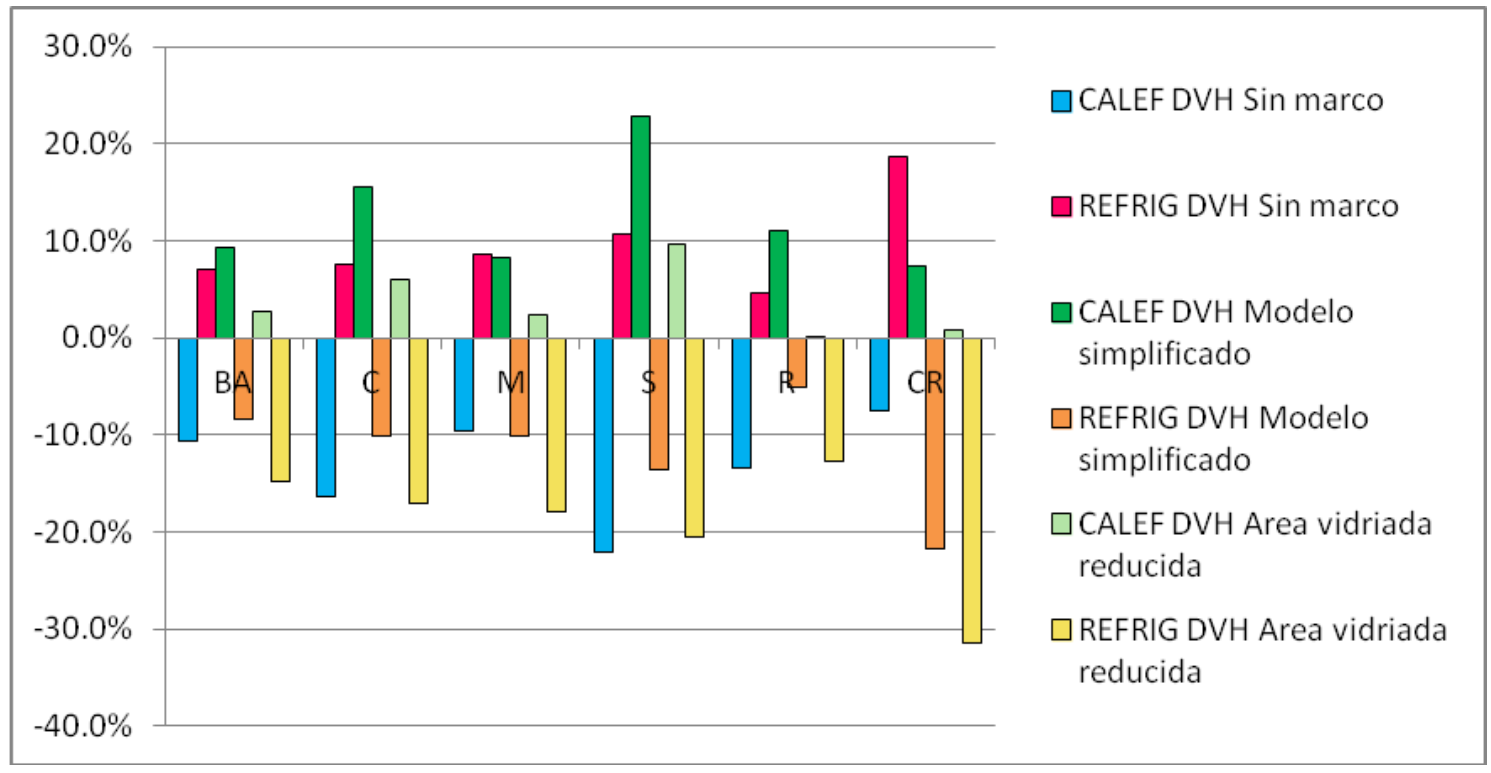

Fuente: Elaboración propia 
Figura 8a. Comparación de las cargas térmicas (caso Buenos Aires)

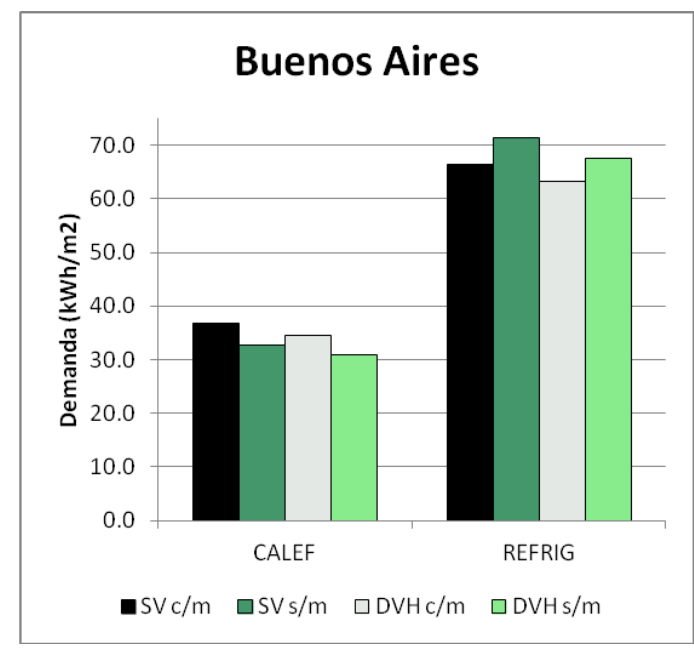

Fuente: Elaboración propia

diados (Figuras 8 a y 8b). En el período de calefacción, el error asociado a la omisión de los marcos en el caso del simple vidriado $(-10,8 \%)$ es mayor a la diferencia de cargas térmicas obtenida entre el caso con SV y DVH (-5,7\%). Asimismo, en el período de refrigeración, el error cometido al no considerar el marco en el caso del DVH (7,0\%), es mayor a la diferencia de demanda obtenida entre los casos con SV y DVH (4,9\%). Estos resultados remarcan la importancia de tomar en cuenta los marcos en el modelado de un edificio en EnergyPlus.

\section{Conclusiones}

El método aconsejable para realizar una simulación edilicia precisa es el modelado detallado del marco, pero este requiere el conocimiento de las características dimensionales y propiedades térmicas de los marcos empleados. Por lo tanto resulta fundamental la caracterización térmica de las aberturas del mercado nacional en función de sus tecnologías y diseños específicos. Esta caracterización puede realizarse con precisión mediante los programas THERM y WINDOW para luego importar los resultados de la simulación a EnergyPlus.

En cuanto al método simplificado, se necesita conocer los índices de transmitancia térmica y factor solar de la ventana completa. Contra-
Figura 8b. Comparación de las cargas térmicas (caso Mendoza).

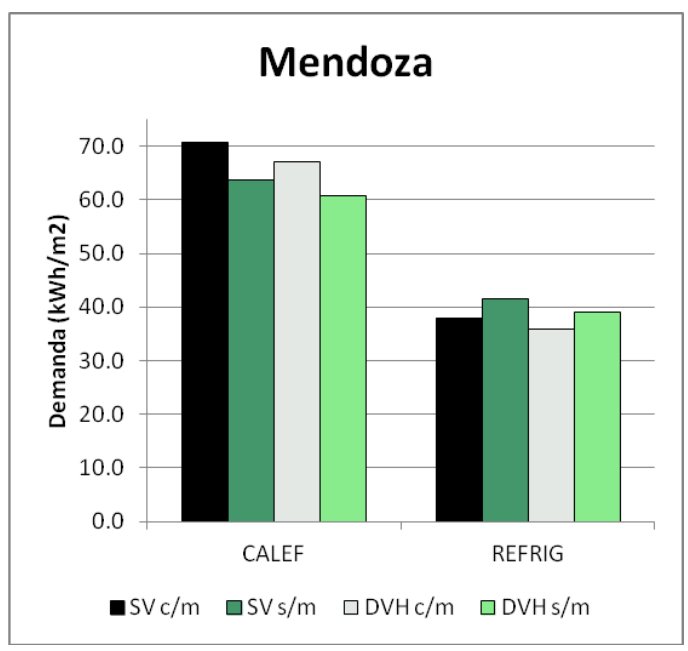

Fuente: Elaboración propia

riamente a otros países donde la implementación de un sistema de etiquetado energético de aberturas ha impulsado la medición y/o cálculo de dichos índices, en Argentina hay una carencia de información para la mayoría de las tecnologías y diseños de ventanas disponibles. En consecuencia, para obtener los índices requeridos, se debe calcular primero los índices asociados al marco, por lo tanto conviene utilizar directamente el método detallado.

Finalmente, el método que omite la existencia del marco es una alternativa frecuentemente elegida debido a la falta de información acerca de las características de los marcos, pero implica errores de hasta un $23 \%$ en la estimación de las cargas de acondicionamiento térmico para el ejemplo de vivienda analizado. El artefacto de reducir las dimensiones de las ventanas para que la superficie vidriada resultante sea igual a la real, tampoco produce resultados satisfactorios (errores de hasta un 32\%). Por más que no se disponga de información suficiente acerca de los marcos de ventanas del edificio a simular, se recomienda de todas formas el modelado de los marcos, aunque sea con valores de conductancia y dimensiones aproximadas, de manera a considerar la reducción de la superficie vidriada resultante de la presencia de las carpinterías opacas, y los intercambios de calor a nivel del marco más elevados que a través de las paredes de la vivienda. 


\section{Bibliografía}

Alchapar, N., Correa, E., \& Lesino, G. (2012). Reflectividad solar de revestimientos horizontales en la envolvente urbana y su capacidad para mitigar la isla de calor. Revista Energías Renovables y Medio Ambiente. 28, pp 37-46

Arasteh, D., Kohler, C., \& Griffith, B. (2009). Modeling windows in Energy Plus with simple performance indices. Lawrence Berkeley National Laboratory, LBNL-2804E, 2009.

Balter, J., Ganem, C., \& Discoli, C. (2012). Auditoría y simulación termoenergética como instrumentos de validación para el estudio y mejoramiento de edificios en altura en "ciudades oasis". Ejemplo de aplicación sobre una unidad de vivienda en la ciudad de Mendoza. Revista Avances en Energías Renovables y Medio Ambiente. 16, pp 05.59-05.66

Barea, G., Esteves, A., Ganem, C., \& Flores Larsen, S. (2011). Evaluación energética de ventanas multiacimutales en la ciudad de Mendoza mediante prototipos a escala 1:1 y el programa EnergyPlus. Revista Avances en Energías Renovables y Medio Ambiente. 15, pp 08.173- 08.181,

De Gastines, M., Villalba, A., \& Pattini, A. (2014). Determinación del Factor K de ventanas. Utilización de programas de simulación de precisión para evaluar el impacto del diseño de marcos y de las condiciones climáticas. Revista Avances en Energías Renovables y Medio Ambiente. Vol. 18, pp 08.35-08.42

De Gastines, M., Correa, E., \& Pattini, A. (2015). Evaluación del balance energético de ventanas en Mendoza. Impacto de su tecnología y orientación. Revista Avances en Energías Renovables y Medio Ambiente. 13, pp 05.01-05.12

Norma IRAM 11507-4:2001. Carpintería de obra y fachadas integrales livianas: Ventanas exteriores: Parte 4: Requisitos complementarios: Aislación térmica. Instituto Argentino de Normalización. Buenos Aires.

Norma ISO 15099:2003. Thermal performance of Windows, Doors and Shading Devices—Detailed Calculations. International Organization for Standardization.

Lyons, P., Wong, J., \& Bhandari, M. (2010). A comparison of window modelling methods in EnergyPlus 4.0. Fourth National Conference of IBPSA-USA, New-York, pp. 177-184.

Monroy, M. (1995). Comportamiento térmico de cerramientos soleados. Tesis Doctoral. Universidad de las Palmas de Gran Canaria, Apéndice B.

Norma NFRC 100-2004. Procedure for Determining Fenestration Product U-Factors. National Fenestration Rating Council, United States.

Winkelmann, F. (2001). Modeling Windows in EnergyPlus. Seventh International Conference IBPSA, Brazil, pp. 457-464. 